\title{
BILINEAR AND BILATERAL GENERATING FUNCTIONS OF GENERALIZED POLYNOMIALS
}

\author{
CH. WALI MOHAMMAD ${ }^{1}$
}

(Received 14 March 1995; revised 15 June 1996)

\begin{abstract}
The paper contains mainly three theorems involving generating functions expressed in terms of single and double Laplace and beta integrals. The theorems, in turn, yield, as special cases, a number of bilinear and bilateral generating functions of generalized functions particularly general double and triple hypergeometric series. One variable special cases of the generalized functions are important in several applied problems.
\end{abstract}

\section{Introduction}

Generalized functions occupy the pride of place in literature on special functions. Their importance which is mounting everyday stems from the fact that they generalize well-known one variable special functions namely, Hermite polynomials, Laguerre polynomials, Legendre polynomials, Gegenbauer polynomials, Jacobi polynomials, Rice polynomials etc. All these polynomials are closely associated with problems of applied nature. For example, Gegenbauer polynomials are deeply connected with axially symmetric potentials in $n$ dimensions and contain the Legendre and Chebyshev polynomials as special cases. The hypergeometric function of which the Jacobi polynomial is a special case, is important in many cases of mathematical analysis and its applications. Further, Bessel functions used in our work are closely associated with problems possessing circular or cylindrical symmetry. For example, they arise in the study of free vibrations of a circular membrane and in finding the temperature distribution in a circular cylinder. They also occur in electromagnetic theory and numerous other areas of physics and engineering.

This paper aims at establishing three theorems involving bilinear and bilateral generating functions of generalized polynomials, expressed in terms of Laplace and beta integrals.

\footnotetext{
${ }^{1}$ Faculty of Engineering and Technology J. M. I. University, New Delhi, INDIA

(C) Australian Mathematical Society, 1997, Serial-fee code 0334-2700/97
} 
Theorem 1 establishes a generating function for generalized triple hypergeometric series $F^{(3)}$ of Srivastava [29, p. 428] expressed in terms of beta transform of one variable. It is worth pointing out that $F^{(3)}$ is a generalization of $F_{1}$ to $F_{14}[16, \mathrm{pp}$. 113114] series of Lauricella, Kampé dé Fériet's double series $F^{(2)}$ [1, p. 150 (29)]; see also [5, p. 112]), $H_{A}, H_{B}$ and $H_{C}$ of Srivastava [28, pp. 99-100], see also [30]), and $F_{K}$ of Sharma [26, p. 613(2)]). Theorem 2 is associated with obtaining a generating function for Appell's function $F_{2}$ expressed in terms of Laplace transform of one variable. Theorem 3 is devoted to proving a generating function for Appell's function $F_{4}$, expressed in terms of a Laplace transform of two variables.

The above theorems yield, as special cases, some known results of Mathur [20], Manocha [17], Manocha and Sharma [19], Sharma and Mittal [27], Saran [25], Carlitz [6] and Halim and Al-Salam [13].

\section{Definitions}

We define hypergeometric functions and polynomials, which we need in our work, as follows:

The Kampé dé Fériet's double hypergeometric function $F^{(2)}[1$, p. 150] is defined as

$$
F^{(2)}\left[\begin{array}{l}
(a):(b) ;(d) ; \\
(f):(g) ;(h) ;
\end{array}, Y\right]=\sum_{m, n=0}^{\infty} \frac{[(a)]_{m+n}[(b)]_{m}[(d)]_{n}}{[(f)]_{m+n}[(g)]_{m}[(h)]_{n}} \frac{x^{m}}{m !} \frac{y^{n}}{n !},
$$

where $(a)$ and $[(a)]_{m+n}$ will mean the sequence of $A$ parameters $a_{1}, a_{2}, \ldots, a_{A}$ and the product $\left(a_{1}\right)_{m+n}\left(a_{2}\right)_{m+n} \cdots\left(a_{A}\right)_{m+n}$ respectively. Thus $[(a)]_{m}$ is to be interpreted as

$$
[(a)]_{m}=\prod_{i=1}^{A}\left(a_{i}\right)_{m}=\left(a_{1}\right)_{m}\left(a_{2}\right)_{m} \cdots\left(a_{A}\right)_{m}=\prod_{i=1}^{A} \frac{\Gamma\left(a_{i}+m\right)}{\Gamma\left(a_{i}\right)},
$$

with similar interpretation for $[(b)],[(d)]$ etc. $F^{(2)}$ has Appell's functions $F_{1}, F_{2}$ and $F_{4}$ as special cases, that is,

$$
\begin{aligned}
& F_{1}[a ; b, c ; d ; X, Y]=\sum_{m, n=0}^{\infty} \frac{(a)_{m+n}(b)_{m}(c)_{n}}{(d)_{m+n}} \frac{x^{m}}{m !} \frac{y^{n}}{n !}, \quad \max \{|X|,|Y|\}<1 \\
& F_{2}[a ; b, c ; d, e ; X, Y]=\sum_{m, n=0}^{\infty} \frac{(a)_{m+n}(b)_{m}(c)_{n}}{(d)_{m}(e)_{n}} \frac{x^{m}}{m !} \frac{y^{n}}{n !}, \quad|X|+|Y|<1 \\
& F_{4}[a, b ; c, d ; X, Y]=\sum_{m, n=0}^{\infty} \frac{(a)_{m+n}(b)_{m+n}}{(c)_{m}(d)_{n}} \frac{x^{m}}{m !} \frac{y^{n}}{n !}, \quad\left|X^{1 / 2}\right|+\left|Y^{1 / 2}\right|<1
\end{aligned}
$$


The generalized hypergeometric function $F^{(3)}$ of 3-variables is defined as

$$
\begin{aligned}
F^{(3)} & {\left[\begin{array}{l}
(a)::(b) ;\left(b^{\prime}\right) ;\left(b^{\prime \prime}\right):(c) ;\left(c^{\prime}\right) ;\left(c^{\prime \prime}\right) ; \\
(d)::(e) ;\left(e^{\prime}\right) ;\left(e^{\prime \prime}\right):(f) ;\left(f^{\prime}\right) ;\left(f^{\prime \prime}\right) ;
\end{array}, Y, Z\right] } \\
& =\sum_{m . n . p=0}^{\infty} \frac{[(a)]_{m+n+p}[(b)]_{m+n}\left[\left(b^{\prime}\right)\right]_{n+p}\left[\left(b^{\prime \prime}\right)\right]_{m+p}[(c)]_{m}\left[\left(c^{\prime}\right)\right]_{n}\left[\left(c^{\prime \prime}\right)\right]_{p}}{[(d)]_{m+n+p}[(e)]_{m+n}\left[\left(e^{\prime}\right)\right]_{n+p}\left[\left(e^{\prime \prime}\right)\right]_{m+p}[(f)]_{m}\left[\left(f^{\prime}\right)\right]_{n}\left[\left(f^{\prime \prime}\right)\right]_{p}} \frac{x^{m}}{m !} \frac{y^{n}}{n !} \frac{z^{p}}{p !},
\end{aligned}
$$

where $(a)$ and $[(a)]_{m}$ have their usual meaning as explained in (2.2) with similar interpretation for $(b),\left(b^{\prime}\right),\left(b^{\prime \prime}\right)$ and so on. It is to be noted here that $A$ is the number of parameters in $(a), B$ is the number of parameters in $(b)$ with similar interpretation for $\left(b^{\prime}\right),\left(b^{\prime \prime}\right)$ and so on.

It will be assumed throughout the paper that the absence of parameters shown by horizontal dashes mean that there exist no parameters, and in that case, from (2.2), the conventional value of an empty product will be unity, that is, $\prod_{i=1}^{0}\left(a_{i}\right)_{m}=1$. Also, numerator parameters like $(a),(b),\left(b^{\prime}\right),\left(b^{\prime \prime}\right)$ etc. may be zero or negative integers, but the denominator parameters like $(d),(e),\left(e^{\prime}\right)$ etc. are not allowed to be zero or negative integers.

The region of convergence of the above triple power series (2.6) is given in the recent literature [8, p. 156]; see also [9, p. 40].

Lauricella's functions $F_{A}^{(n)}$ and $F_{C}^{(n)}$ of $n$ variables in the notation of Lauricella [16] are as follows.

$$
\begin{gathered}
F_{A}^{(n)}\left[a, b_{1}, b_{2}, \ldots, b_{n} ; c_{1}, \ldots, c_{n} ; x_{1}, \ldots, x_{n}\right] \\
=\sum_{m_{1}, \ldots, m_{n}=0}^{\infty} \frac{(a)_{m_{1}+\cdots+m_{n}}\left(b_{1}\right)_{m_{1}} \cdots\left(b_{n}\right)_{m_{n}}}{\left(c_{1}\right)_{m_{1}} \cdots\left(c_{n}\right)_{m_{n}}} \frac{x_{1}^{m_{1}}}{m_{1} !} \cdots \frac{x_{n}^{m_{n}}}{m_{n} !} \\
F_{C}^{(n)}\left[a, b ; c_{1}|+| x_{2}|+\cdots+| x_{n} \mid<l ;\right. \\
=\sum_{m_{1}, \ldots, m_{n}=0}^{\infty} \frac{(a)_{m_{1}+\cdots+m_{n}}(b)_{m_{1}+\cdots+m_{n}}}{\left(c_{1}\right)_{m_{1}} \cdots\left(c_{n}\right)_{m_{n}}} \frac{x_{1}^{m_{1}}}{m_{1} !} \cdots \frac{x_{n}^{m_{n}}}{m_{n} !} \\
\left|\sqrt{x_{1}}\right|+\left|\sqrt{x_{2}}\right|+\cdots+\left|\sqrt{x_{n}}\right|<l .
\end{gathered}
$$

For $n=2$, equations (2.7) and (2.8) reduce to Appell's functions $F_{2}$ and $F_{4}$ respectively.

The functions $F_{E}$ and $F_{G}$ in the notation of Saran [23], indicating also the numbering 
of Lauricella's [16] on the left are as follows:

$$
\begin{aligned}
& F_{4}: F_{E} {[a, a, a, b, c, c ; d, e, f ; x, y, z] } \\
&= \sum_{m, n, p=0}^{\infty} \frac{(a)_{m+n+p}(b)_{m}(c)_{n+p}}{(d)_{m}(e)_{n}(f)_{p}} \frac{x^{m}}{m !} \frac{y^{n}}{n !} \frac{z^{p}}{p !}, \quad r+\left(s^{1 / 2}+t^{1 / 2}\right)=1 ; \\
& F_{8}: F_{G}[a, a, a ; b, c, d ; e, f, f ; X, Y, Z] \\
&=\sum_{m, n, p=0}^{\infty} \frac{(a)_{m+n+p}(b)_{m}(c)_{n}(d)_{p}}{(e)_{m}(f)_{n+p}} \frac{x^{m}}{m !} \frac{y^{n}}{n !} \frac{z^{p}}{p !}, \quad r+s=1+r+t .
\end{aligned}
$$

The Jain's functions $3 \varphi_{A}^{(1)}$ and $3 \varphi_{G}^{(1)}[14]$ are as defined below.

$$
\begin{aligned}
3 \varphi_{A}^{(1)} & {\left[a, b_{1}, b_{2} ; c_{1}, c_{2}, c_{3} ; X, Y, Z\right] } \\
= & \sum_{m, n, p=0}^{\infty} \frac{(a)_{m+n+p}\left(b_{1}\right)_{m}\left(b_{2}\right)_{n}}{\left(c_{1}\right)_{m}\left(c_{2}\right)_{n}\left(c_{3}\right)_{p}} \frac{x^{m}}{m !} \frac{y^{n}}{n !} \cdot \frac{z^{p}}{p !}, \\
3 \varphi_{G}^{(1)} & {\left[a, a, a, b_{1}, b_{2} ; c_{1}, c_{2}, c_{2} ; X, Y, Z\right] } \\
& =\sum_{m, n, p=0}^{\infty} \frac{(a)_{m+n+p}\left(b_{1}\right)_{m}\left(b_{2}\right)_{n}}{\left(c_{1}\right)_{m}\left(c_{2}\right)_{n+p}} \frac{x^{m}}{m ! n !} \frac{y^{n}}{n !} \frac{z^{p}}{p !} .
\end{aligned}
$$

The generalized Rice polynomial [15, p. 158], defined as

$$
H_{n}^{(\alpha, \beta)}(p, q, x)=\frac{(1+\alpha)_{n}}{n !}{ }_{3} F_{2}[-n, n+\alpha+\beta+1, p ; 1+\alpha, q ; X],
$$

has the Jacobi polynomial $P_{n}^{(\alpha, \beta)}(X)$ [22, p. 254], the generalized Laguerre polynomial $L_{n}^{\alpha}(X)\left[22\right.$, p. 200] and the Gegenbauer polynomials $C_{n}^{\alpha}(X)$ as special cases defined as

$$
\begin{gathered}
H_{n}^{(\alpha, \beta)}\left(p, p, \frac{1-x}{2}\right)=P_{n}^{(\alpha, \beta)}(X) \\
=\frac{(1+\alpha)_{n}}{n !}{ }_{2} F_{1}\left[-n, 1+\alpha+\beta+n ; 1+\alpha ; \frac{1-X}{2}\right], \\
P_{n}^{(\alpha, \alpha)}(X)=C_{n}^{\alpha}(X)=\frac{(1+\alpha)_{n}}{n !}{ }_{2} F_{1}\left[-n, 1+2 \alpha+n ; \alpha+1 ; \frac{1-X}{2}\right], \\
L_{n}^{\alpha}(X)=\frac{(1+\alpha)_{n}}{n !}{ }_{1} F_{1}[-n ; 1+\alpha ; X],
\end{gathered}
$$

where in each of the equations (2.13) to (2.16) $\operatorname{Re}(\alpha)>-1, \operatorname{Re}(\beta)>-1$ and $n$ being a non-negative integer.

The Laguerre polynomials play an important role in finding the wave function associated with the electron in a hydrogen atom. Further, Laguerre polynomials are 
encountered in the solution of the problem on the propagation of electromagnetic waves in long lines and in the analysis of the motion of electrons in Coulomb field, as well as in certain other problems.

The Bediant polynomial $R_{n}(\alpha, \beta ; X)[3$, p. 15] is defined as

$$
R_{n}(\alpha, \beta ; X)=\frac{(\alpha)_{n}(2 X)^{n}}{n !}{ }_{3} F_{2}\left[-\frac{n}{2},-\frac{n}{2}+\frac{1}{2}, \beta-\alpha ; \beta, 1-\alpha-n ; \frac{1}{X^{2}}\right] .
$$

The generalized Sylvester polynomial $f_{n}(x ; a)$ is defined as

$$
f_{n}(x ; a)=\frac{(a x)^{n}}{n !}{ }_{2} F_{0}\left[-n, X ;-;-\frac{1}{a x}\right],
$$

where $a \neq 0$ is an arbitrary constant.

The Lagrange's polynomial $g_{n}^{\alpha, \beta}(X, Y)[10$, p. 267] is defined as

$$
g_{n}^{\alpha . \beta}(X, Y)=\sum_{r=0}^{\infty} \frac{(\alpha)_{r}(\beta)_{n-r}}{r !(n-r) !} x^{r} y^{n-r} .
$$

The Bessel function $J_{n}(x)$ of order $n$ in $x$ is defined as

$$
J_{n}(x)=\frac{(x / 2)^{n}}{\Gamma(n+1)}{ }_{0} F_{1}\left[\begin{array}{c}
-; \\
n+1 ;
\end{array}-\frac{x^{2}}{4}\right],
$$

where $-\infty<x<\infty$ and $n$ is a positive integer or zero.

\section{Main theorems}

THEOREM 1. Let $F(x, t)$ be a function having a formal power series expansion in $t$, given by

$$
F(x, t)=\sum_{n=0}^{\infty} C_{n} f_{n}(x) t^{n}
$$

where $C_{n}$ is a specified sequence of parameters, independent of $x$ and $t$, and $f_{n}(x)$, $n=0,1,2, \ldots$, are polynomials of degree $n$ in $x$. Then, for $\operatorname{Re}(q)>0, \operatorname{Re}(p)>0$, $p$ and $q$ being complex numbers, and with restrictions on $x$ 's and $t$, such that the triple hypergeometric series of Srivastava and $F\left(x, \frac{t z}{z-1}\right)$ remain uniformly convergent for $z \in(0,1)$,

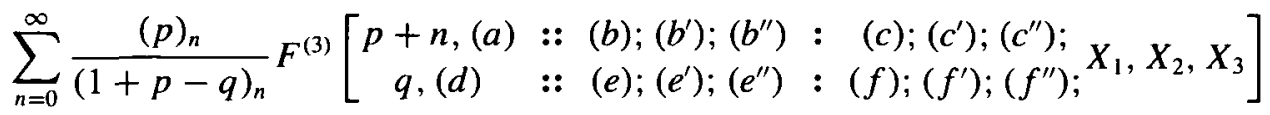




$$
\begin{aligned}
& f_{n}(x) t^{n}=K \int_{0}^{1} z^{p-1}(1-z)^{q-p-1} \\
& \times F^{(3)}\left[\begin{array}{c}
(a)::(b) ;\left(b^{\prime}\right) ;\left(b^{\prime \prime}\right):(c) ;\left(c^{\prime}\right) ;\left(c^{\prime \prime}\right) ; \\
(d)::(e) ;\left(e^{\prime}\right) ;\left(e^{\prime \prime}\right):(f) ;\left(f^{\prime}\right) ;\left(f^{\prime \prime}\right) ;
\end{array} X_{1} Z, X_{2} Z, X_{3} Z\right] F\left(x, \frac{t z}{z-1}\right) d z
\end{aligned}
$$

where $K=\frac{\Gamma(q)}{\Gamma(p) \Gamma(q-p)}$, $(a)_{n}$ is the Pochhammer symbol, defined by

$$
(a)_{n}=\frac{\Gamma(a+n)}{\Gamma(a)}=\left\{\begin{array}{l}
n ! \quad \text { if } a=1 \text { and } n \geq 1 \\
1, \quad \text { if } n=0 \\
\prod_{K=1}^{n}(a+K-1)=a(a+1) \cdots(a+n-1) \text { for } n \geq 1
\end{array}\right.
$$

and $F^{(3)}[X, Y, Z]$ is the triple series, defined by (2.6).

THEOREM 2. Let

$$
F(x, t)=\sum_{n=0}^{\infty} f_{n}(x) t^{n}
$$

where $f_{n}(x)$ is a polynomial of degree $n$ in $x$. Then for Appell's function $F_{2}$ (see equation (2.4))

$$
\begin{aligned}
& \frac{1}{\Gamma(b)} \int_{0}^{1} e^{-p} p^{b-1}{ }_{1} F_{1}[c ; b ; Y p]_{1} F_{1}[a ; d ; Z p] d p \\
& \quad=\sum_{n=0}^{\infty}(b)_{n} F_{2}[b+n ; a, c ; d, b ; Z, Y] f_{n}(x) t^{n}
\end{aligned}
$$

provided the integral is convergent.

THEOREM 3. Let

$$
F(x, t)=\sum_{n=0}^{\infty} f_{n}(x) t^{n}
$$

where $f_{n}(x)$ is a polynomial of degree $n$ in $x$. Then

$$
\begin{gathered}
\frac{1}{\Gamma(a) \Gamma(b)} \int_{0}^{\infty} \int_{0}^{\infty} e^{-(p+q)} p^{a-1} q^{b-1}{ }_{0} F_{1}[-; c ; Y p q]_{0} F_{1}[-; d ; Z P q] F(x, t p q) d p d q \\
\quad=\sum_{n=0}^{\infty}(a)_{n}(b)_{n} F_{4}[a+n, b+n ; c, d ; Y, Z] f_{n}(x) t^{n}
\end{gathered}
$$

provided the integral is convergent. 
Proof of TheOREMS 1 To 3. To prove Theorem l, we replace $F(x, t)$ by its power series (3.1) and $F^{(3)}[X, Y, Z]$ by its series representation (2.6) in the integrand of (3.2). Changing the order of integration and summation, which is permissible due to the uniform convergence of the series involved and evaluating the inner beta function integral, we arrive at the result (3.2).

The proofs of Theorems 2 and 3 are similar to that of Theorem 1 .

On taking $B=B^{\prime}=B^{\prime \prime}=E=E^{\prime \prime}=0$ (Here $B, B^{\prime}, B^{\prime \prime}$ etc. are the number of parameters in $(b),\left(b^{\prime}\right),\left(b^{\prime \prime}\right)$ and so on), $X_{3}=0$, Theorem 1 gives an elegant result in the form

$$
\begin{aligned}
& \sum_{n=0}^{\infty} \frac{(p)_{n}}{(1+p-q)_{n}} F^{(2)}\left[\begin{array}{c}
p+n,(a):(c),\left(c^{\prime}\right) ; \\
q,(d):(f),\left(f^{\prime}\right) ;
\end{array} X_{1}, X_{2}\right] f_{n}(x) t^{n} \\
& =K \int_{0}^{1} Z^{p-1}(1-Z)^{q-p-1} F^{(2)}\left[\begin{array}{c}
(a):(c),\left(c^{\prime}\right) ; \\
(d):(f),\left(f^{\prime}\right) ;
\end{array} X_{1} Z, X_{2} Z\right] F\left(x, \frac{t z}{z-1}\right) d z,
\end{aligned}
$$

where $F^{(2)}\left[X_{1}, X_{2}\right]$ is defined by $(2.1)$.

On taking $B=B^{\prime}=B^{\prime \prime}=E=E^{\prime}=E^{\prime \prime}=D=0, C=C^{\prime}=C^{\prime \prime}=F=F^{\prime}=$ $F^{\prime \prime}=A=1$, then replacing $a_{1}$ with $q$ in Theorem 1 , we get a known result of Mathur [20, p. $222(2.2)]$ :

$$
\begin{aligned}
\sum_{n=0}^{\infty} & \frac{(p)_{n}}{1+p-q} F_{A}\left[p+n, C_{1}, C_{2}, C_{3} ; f_{1}, f_{2}, f_{3} ; X_{1}, X_{2}, X_{3}\right] f_{n}(X) t^{n} \\
= & K \int_{0}^{1} Z^{p-1}(1-Z)^{q-p-1} F_{A}\left[q, C_{1}, C_{2}, C_{3} ; f_{1}, f_{2}, f_{3} ; X_{1} Z, X_{2} Z, X_{3} Z\right] \\
& \quad \times F\left(x, \frac{t z}{z-1}\right) d z
\end{aligned}
$$

Similarly results for Lauricella's functions $F_{B}, F_{C}$ and $F_{D}[12, \mathrm{p} .41]$ given by Mathur [20, pp. 222-223, eqns (2.3) to (2.5)] follow as special cases of Theorem 1.

\section{Applications}

In view of the importance and usefulness of Theorems 1 to 3 , we first present some interesting applications of Theorem 1 .

(i) Consider the generating function for the generalized Rice polynomial [18, p. 432(7)]:

$$
\sum_{n=0}^{\infty} \frac{(\lambda)_{n}}{(-\alpha-\beta)_{n}} H_{n}^{(\alpha-n, \beta-n)}(\xi, j, X) t^{n}=F_{2}[\lambda,-\alpha, \xi ;-\alpha-\beta, j ;-t, X t]
$$


where $H_{n}^{(\alpha, \beta)}(\xi, j, X)$ is the generalized Rice polynomial, defined by (2.13).

In (3.1) we take

$$
F(x, t)=F_{2}(\lambda,-\alpha, \xi ;-\alpha-\beta, j ;-t, x t),
$$

Combining (4.1) with (3.1), we get

$$
\begin{aligned}
& \sum_{n=0}^{\infty} \frac{(p)_{n}}{(\alpha-\beta)_{n}} F^{(2)}\left[\begin{array}{c}
p+n,(a):(c) ;\left(c^{\prime}\right) ; X_{1}, X_{2} \\
q,(d):(f) ;\left(f^{\prime}\right) ; H_{n}^{(\alpha-n, \beta-n)}(\xi, j, X) t^{n}
\end{array}\right. \\
& \quad=\sum_{S=0}^{\infty} \frac{(p)_{S}(\xi)_{S}(x t)^{S}}{(j)_{S} s !} F^{(3)}\left[\begin{array}{c}
p+s::-;(a) ;-:-\alpha ;(c) ;\left(c^{\prime}\right) ; \\
-::-; q,(d) ;-:-\alpha-\beta ;(f),\left(f^{\prime}\right) ;
\end{array}-t, X_{1}, X_{2}\right] .
\end{aligned}
$$

By means of (2.14), (2.15), and (2.16), (4.2) reduces to generating functions involving Jacobi, Gegenbauer and Laguerre polynomials.

The Rice polynomial $H_{n}^{(\alpha-n, \beta-n)}(\xi, j, x)$ generalizes the modified Jacobi polynomials $P_{n}^{(\alpha-n, \beta-n)}(x)$, which, in turn, generalizes the Legendre polynomial. The Legendre polynomials are closely associated with physical phenomena for which spherical geometry is important.

Again, Kampé dé Fériet's function $F^{(2)}$ in (4.2), can be specialized to yield the generating functions for Appell's functions $F_{1}$ to $F_{4}$.

For example, on taking $A=D=F=F^{\prime}=0, C=C^{\prime}=1$, then replacing $\xi$ by $j, X$ by $\frac{1}{2}(1-X)$ equation (4.2) reduces to a result for Jacobi polynomials:

$$
\begin{aligned}
\sum_{n=0}^{\infty} \frac{(p)_{n}}{(-\alpha-\beta)_{n}} & F_{1}\left[p+n, \beta_{1}, \beta_{2} ; q ; Y, Z\right] P_{n}^{(\alpha-n, \beta-n)}(X) t^{n} \\
= & \left(1-\left(\frac{1-x}{2}\right) t\right)^{-p} F_{G}\left[p, p, p,-\alpha, \beta_{1}, \beta_{2} ;-\alpha-\beta, q, q\right. \\
& \left.\frac{-2 t}{2-(1-x) t}, \frac{2 y}{2-(1-x) t}, \frac{2 z}{2-(1-x) t}\right]
\end{aligned}
$$

where $F_{G}$ is given by $(2.10)$.

On taking $A=C=C^{\prime}=F=F^{\prime}=1, D=0$, and then replacing $a_{1}$ by $q$ equation (4.2), reduces in view of (2.14) to

$$
\begin{aligned}
& \sum_{n=0}^{\infty} \frac{(p)_{n}}{(-\alpha-\beta)_{n}} F_{2}\left[p+n, \beta_{1}, \beta_{2} ; \gamma_{1}, \gamma_{2} ; Y, Z\right] P_{n}^{(\alpha-n, \beta-n)}(X) t^{n} \\
& \quad=(\omega)^{-p} F_{A}\left[p, \beta_{1}, \beta_{2},-\alpha ; \gamma_{1}, \gamma_{2} ;-\alpha-\beta ; \frac{Y}{\omega}, \frac{Z}{\omega},-\frac{t}{\omega}\right],
\end{aligned}
$$

where, for convenience we put

$$
\omega=\left(1-\frac{1}{2}(1-X) t\right) .
$$


Similarly, on taking $C=C^{\prime}=D=0, F=F^{\prime}=1, A=2$ and letting one of the $A$ 's parameters be $q,(4.2)$ reduces to

$$
\begin{aligned}
& \sum_{n=0}^{\infty} \frac{(p)_{n}}{(-\alpha-\beta)_{n}} F_{4}\left[p+n, \beta ; \gamma_{1}, \gamma_{2} ; Y, Z\right] P_{n}^{(\alpha-n, \beta-n)}(X) t^{n} \\
& \quad=(\omega)^{-p} F_{E}\left[p, p, p,-\alpha, \beta, \beta ;-\alpha-\beta, \gamma_{1}, \gamma_{2} ;-\frac{t}{\omega}, \frac{Y}{\omega}, \frac{Z}{\omega}\right],
\end{aligned}
$$

where $F_{E}$ is Lauricella's $F_{4}$ (see equation (2.9)).

The results (4.4) and (4.5) are the known results of Manocha [17, p. 457(2.2)] and Sharma and Mittal [27, p. 691(10)] respectively. Some more bilinear and bilateral generating functions involving Jacobi polynomial follow immediately from results (4.3) to (4.5) upon reducing $F_{1}, F_{2}$ and $F_{4}$ to the Gauss function ${ }_{2} F_{1}$.

(ii) Consider the generating function [31, p. 185] (see also [22, p. 302])

$$
\sum_{n=0}^{\infty}(\alpha)_{n} f_{n}(x ; a) t^{n} \cong(1-a x t)^{-\alpha}{ }_{2} F_{0}\left[\alpha, x ;-; \frac{t}{1-a x t}\right],
$$

where $f_{n}(x ; a)$ is the generalized Sylvester polynomial given by $(2.18)$.

On taking $\lambda=1+p-q$ in (4.6) and utilizing the result (3.7) with $X_{2}=0$, we obtain

$$
\begin{aligned}
& \sum_{n=0}^{\infty}(p)_{n 1+A+C} F_{1+D+F}\left[\begin{array}{c}
p+n,(a),(c) ; \\
q,(d),(f) ;
\end{array} X_{1}\right] f_{n}(x ; a) t^{n} \\
& \cong \sum_{m, r, s=0}^{\infty} \frac{(p)_{m+r+s}[(a)]_{m}[(c)]_{m}}{(q)_{m}[(d)]_{m}[(f)]_{m}} \frac{X_{1}^{m}}{m !} \frac{(a x t)^{r}}{r !} \frac{t^{s}}{s !} .
\end{aligned}
$$

Now, multiplying the above by $e^{K} K^{-b}$, replacing $t$ by $t / K$ and then evaluating the result obtained with the help of Hankel's contour integral for the gamma function [12, p. 32(1.5.1.5)]:

$$
\frac{1}{2 \pi i} \int_{C} e^{t} t^{-a-m} d t=\frac{1}{\Gamma(a+m)},
$$

where $m$ is a non-negative integer and $a$ does not take non-positive integer values, we ,get

$$
\begin{aligned}
& \sum_{n=0}^{\infty} \frac{(p)_{n}}{(b)_{n}}{ }_{1+A+C} F_{1+D+F}\left[\begin{array}{c}
p+n,(a),(c) ; \\
q,(d),(f) ;
\end{array} X_{1}\right] f_{n}(x ; a) t^{n} \\
& \quad \cong F^{(3)}\left[\begin{array}{c}
p::-;-;-:(a),(c) ;-; x ; \\
-::-; b ;-: q,(d),(f) ;-;-;
\end{array} \quad X_{1}, a x t, t\right] .
\end{aligned}
$$


Setting $A=C=D=1, F=0$, then replacing $c_{1}$ by $q$ in (4.9), we get after suitable adjustment of parameters and variables that

$$
\sum_{n=0}^{\infty} \frac{(p)_{n}}{(b)_{n}}{ }_{2} F_{1}[p+n, \beta ; \mu ; Y] f_{n}(x ; a) t^{n} \cong 3 \varphi_{G}^{(1)}[p, p, p ; \beta, X ; \mu, b, b ; Y, X t, t]
$$

where $3 \varphi_{G}^{(1)}$ is defined by (2.12).

Again the generalized Sylvester polynomial $f_{n}(X ; a)$ in $(4.10)$ in view of the relation

$$
f_{n}(X ; 1)=\phi_{n}(X)=\frac{X^{n}}{n !}{ }_{2} F_{0}\left[-n, X ;-\frac{1}{X}\right]
$$

reduces to the Sylvester polynomial $\varphi_{n}(X)$ and we get a known result due to Mathur [20, p. 226(3.22)].

(iii) Consider the generating function [31, p. 186] (see also [3])

$$
\sum_{n=0}^{\infty} \frac{(\alpha)_{n}}{(\mu)_{n}} R_{n}(\beta, \mu ; X) t^{n}=F_{2}(\alpha, \beta, \beta ; \mu, \mu ; u t, v t),
$$

where $u=X-\sqrt{x^{2}-1}, v=X+\sqrt{x^{2}-1}$ and $R_{n}(\beta, \mu ; X)$ is the Bediant polynomial, defined by (2.17).

In (3.3), we take $F(X, t)=F_{2}(\alpha, \beta, \beta ; \mu, \mu ; u t, v t)$. Combining (4.11) with (3.3) in Theorem 2, we get

$$
\begin{aligned}
& \sum_{n=0}^{\infty} \frac{(b)_{n}(\alpha)_{n}}{(\mu)_{n}} F_{2}(b+n, a, c ; d, b ; Z, Y) R_{n}(\beta, \mu, X) t^{n} \\
& \quad=\sum_{m, n, r, s=0}^{\infty} \frac{(b)_{m+n+r+s}(c)_{m}(a)_{n}(\alpha)_{r+s}(\beta)_{r}(\beta)_{s}}{(b)_{m}(d)_{n}(\mu)_{r}(\mu)_{s}} \frac{Y^{m}}{m !} \frac{Z^{n}}{n !} \frac{(u t)^{r}}{r !} \frac{(v t)^{s}}{s !}
\end{aligned}
$$

which on replacing $t$ by $t / K$, multiplying by $e^{K} K^{-\alpha}$ and evaluating with the help of the integral (4.8), gives

$$
\begin{gathered}
\sum_{n=0}^{\infty} \frac{(b)_{n}}{(\mu)_{n}} F_{2}(b+n, a, c ; d, b ; Z, Y) R_{n}(\beta, \mu ; X) t^{n} \\
=F_{4}^{(A)}[b, a, c, \beta, \beta ; \alpha, b, \mu, \mu ; Z, Y, u t, v t] .
\end{gathered}
$$

In (4.12), changing $Z$ into $Z / a$ and letting $a \rightarrow \infty$, taking $Y=0$, we get a result for Bediant polynomial involving Jain's function $3 \varphi_{A}^{(1)}$ :

$$
\sum_{n=0}^{\infty} \frac{(b)_{n}}{(\mu)_{n}}{ }_{1} F_{1}[b+n ; d ; Z] R_{n}(\beta, \mu ; X) t^{n}=3 \varphi_{A}^{(1)}[b, b, b, \beta, \beta ; d, \mu, \mu ; Z, u t, v t] .
$$


(iv) Consider the Bateman's generating function [22, p. 70] (see also [2])

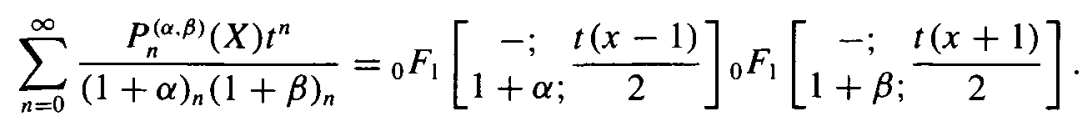

Then for

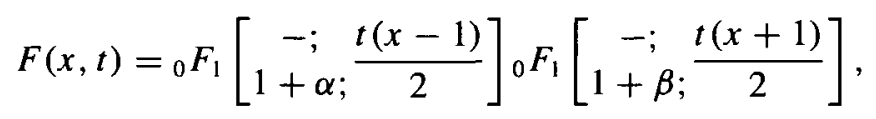

the application of Theorem 3 would give us

$$
\begin{aligned}
& \sum_{n=0}^{\infty} \frac{(a)_{n}(b)_{n}}{(1+\alpha)_{n}(1+\beta)_{n}} F_{4}[a+n, b+n ; c, d ; Y, Z] P_{n}^{(\alpha, \beta)}(X) t^{n} \\
& \quad=F_{C}^{(4)}\left[a, b ; c, d, 1+\alpha, 1+\beta ; Y, Z, \frac{(X-1) t}{2}, \frac{(X+1) t}{2}\right] .
\end{aligned}
$$

On taking $a=\lambda, b=\delta, c=1+\mu$ and $Z=0$ in (4.14), we get a known result of Manocha and Sharma [19, p. 79(31)]

$$
\begin{aligned}
& \sum_{n=0}^{\infty} \frac{(\lambda)_{n}(\delta)_{n}}{(1+\alpha)_{n}(1+\beta)_{n}}{ }_{2} F_{1}[\lambda+n, \lambda+n ; 1+\mu, Y] P_{n}^{(\alpha, \beta)}(X) t^{n} \\
& \quad=F_{C}^{(3)}\left[\lambda, \delta ; 1+\alpha, 1+\beta, 1+\mu ; \frac{(X-1) t}{2}, \frac{(X+1) t}{2}, Y\right]
\end{aligned}
$$

which was originally obtained by a fractional derivative technique.

When we put $C=1+\alpha, d=1+\beta$ and $Y=Z=0$, equation (4.14) yields a result due to Brafman [4, p. 943].

Again when we put $Z=0$, replace $y$ by $y /(y-1), t$ by $t /(1-y), C$ by $a, d$ by $b$ and use Euler transformation [22, p. 60(4)] we get a known result of Saran [25, p. 16 (3.3)].

It may be pointed out that ${ }_{0} F_{1}$ defines a Bessel function. Bessel functions are common in the solution of problems featuring circular or cylindrical domains.

(v) Consider the generating relation [31, p. 85(25)] (see also [11, p. 267])

$$
(1-x t)^{-\alpha}(1-y t)^{-\beta}=\sum_{n=0}^{\infty} g_{n}^{\alpha, \beta}(X, Y) t^{n},
$$

where $g_{n}^{\alpha, \beta}(X, Y)$ is the Lagrange polynomial, defined by (2.19).

In (3.5), we take $F(x, t)=(1-x t)^{-\alpha}(1-y t)^{-\beta}$ combine (4.16) with (3.5) and then in the result obtained replace $t$ by $t / p q$. Multiplying by $e^{p+q}(p)^{-c}(q)^{-d}$ and 
evaluating with the help of the integral (4.8), we obtain

$$
\begin{aligned}
& \sum_{n=0}^{\infty} \frac{(a)_{n}(b)_{n}}{(c)_{n}(d)_{n}} F_{4}[a+n, b+n ; a, b ; Y, Z] g_{n}^{(\alpha, \beta)}(X, Y) t^{n} \\
& \quad=\sum_{m=0}^{\infty}(b)_{m} \frac{(Y)^{m}}{m !} F^{(3)}\left[\begin{array}{c}
a+m, b+m::-;-;-:-; \alpha ;-\beta ; \\
-::-; c, d ;-: b ;-;-;
\end{array}\right.
\end{aligned}
$$

Since on replacing $x$ by $-(1+x) / 2, y$ by $(1-x) / 2, \alpha$ by $-\alpha$ and $\beta$ by $-\beta$, (4.16) reduces to a result of Carlitz [6] (see also [31, p. 82(2)]) involving the Jacobi polynomial $P_{n}^{\alpha-n, \beta-n}(X)$, the above replacement in (4.17) offers us

$$
\begin{aligned}
\sum_{n=0}^{\infty} & \frac{(a)_{n}(b)_{n}}{(c)_{n}(d)_{n}} F_{4}[a+n, b+n ; a, b ; Y, Z] P_{n}^{\alpha-n . \beta-n}(X) t^{n} \\
= & \sum_{m=0}^{\infty}(b)_{m} \frac{(Y)^{m}}{m !} \\
& \times F^{(3)}\left[\begin{array}{c}
a+m, b+m,::-;-;-:-; \alpha ;-\beta ; \\
-::-; c, d ;-: b ;-;-;
\end{array}\right.
\end{aligned}
$$

which on taking $Z=0$, replacing $y$ by $y /(y-1), t$ by $t /(y-1)$ and making use of Euler's transformation, gives a result due to Saran [25, p. 16 (3.4)]:

$$
\begin{aligned}
& \sum_{n=0}^{\infty} \frac{(a)_{n}(b)_{n}}{(c)_{n}(d)_{n}}{ }_{2} F_{1}[-n, b+n ; a ; Y] P_{n}^{(\alpha-n, \beta-n)}(X) t^{n} \\
& \quad=(1-y)^{-b} F^{(3)}\left[\begin{array}{c}
a, b,::-;-;-:-;-\alpha ;-\beta ; \frac{Y}{Y-1}, \frac{1(X+1) t}{2(Y-1)}, \frac{1(X+1) t}{2(Y-1)} \\
-:: c, d ;-: a ;-;-;
\end{array}\right.
\end{aligned}
$$

Also, equation (4.19) is a generalization of known results [31, p. 105 (eqns. (4) and (7)), p. 106 (eqns. (8) to (11) and p. 107 (eqn. (12)]

\section{Acknowledgement}

I wish to thank the referee for his valuable suggestions that resulted in a much improved presentation of this paper. 


\section{References}

[1] P. Appell and J. Kampé dé Fériet's, Fonctions hypergèometriques et hypersphériques: Polynomes d'Hermite (Gauthier-Villars et cie, Paris, 1926).

[2] H. Bateman, "A generalization of Legendre polynomial", Proc. Lond. Math. Soc. Series (2) 3 (1905) 111-123.

[3] P. E. Bedient, "Polynomials related to Appell's functions of two variables", Ph. D. Thesis, Univirsity of Michigan, 1959.

[4] F. Brafman, "Generating function of Jacobi and related polynomials", Proc. Amer. Math. Soc. 2 (1951) 942-949.

[5] J. L. Burchall and T. W. Chaundy, "Expansions of Appell's double hypergeometric functions (II)", Quart. J. Math. Oxford Ser. 12 (1941) 112-128.

[6] L. Carlitz, "A bilinear generating function for the Jacobi polynomials", Bull. Un. Math. Ital. (3) 18 (1963) 87-89.

[7] Chaudhary Wali Mohd. and M. I. Qureshi, "Expansion formulae for general triple hypergeometric series”, J. Austral. Math. Soc. Ser. B 27 (1986) 376-385.

[8] S. P. Chhabra and K. C. Rusia, "A transformation formula for a general hypergeometric function of three variables", Jñänäbha 9/10 (1980) 155-159.

[9] V. L. Deshpande, "Certain formulas associated with hypergeometric functions of three variables", Pure and Applied Mathematics Science 14 (1981) 39-45.

[10] A. Erdélyi, Higher Transcendental Functions Vol. I (Bateman manuscript Project McGraw-Hill Book Co. Inc., New York, Toronto and London, 1953).

[11] A. Erdélyi, Higher Transcendental Functions Vol. III (Bateman manuscript Project McGraw-Hill Book Co. Inc., New York, Toronto and London, 1955).

[12] H. Exton, Multiple hypergeometric functions and applications (John Wiley and Sons (Halsted Press), New York, Ellis Horwood, Chichester, 1976).

[13] N. A. Halim and W. A. Al-Salam, "Double Euler transformations of certain hypergeometric functions", Duke Math. J. 30 (1963) 51-62.

[14] R. N. Jain, "The confluent hygergeometric functions of three variables", Proc. Nat. Acad. Sci. India Sect. A 36(2) (1966) 395-408.

[15] P. R. Khandekar, "On a generalization of Rice polynomial", Proc. Nat. Acad. Sci. India Sect. A 34(2) (1964) 157-162.

[16] G. Lauricella, "Sulle funzioni ipergeometriche a piú variabili", Rend. Circ. Mat. Palermo 7 (1893) 111-158.

[17] H. L. Manocha, "Some bilinear generating functions for Jacobi polynomials", Proc. Camb. Philos. Soc. 63 (1967) 457-459.

[18] H. L. Manocha, "Some formulae for generalized Rice polynomials", Proc. Camb. Philos. Soc. 64 (1968) $431-434$.

[19] H. L. Manocha and B. L. Sharma, "Infinite series of hypergeometric functions", Ann. Soc. Sci. Bruxelles Ser. 180 (1966) 73-86.

[20] B. L. Mathur, “On some results involving Lauricella functions”, Bull. Cal. Math. Soc. 70 (1978) 221-227.

[21] P. C. Munot, B. L. Mathur and R. S. Kushwaha, "On generating functions for classical polynomials", Proc. Nat. Acad. Sci. India Sect. A 45 (1975) 187-192.

[22] E. D. Rainville, Special functlons, (MacMillan Co., New York, 1960), reprinted by Chelsea Publ. Co., Bronx, New York, 1971.

[23] S. Saran, "Hypergeometric functions of three variables", Ganita 5 (1954) 71-91, Corrigendum. Ibid 7 (1956), 65.

[24] S. Saran, "A general theorem for bilinear generating functions", Pacific J. Math. 35 (1970) 783 
786.

[25] S. Saran, "Theorems on bilinear generating functions", Indian J. Pure Appl. Math. 3(1) (1972) $12-20$.

[26] B. L. Sharma, "Some theorems for Appell's functions", Proc. Camb. Philos. Soc. 67 (1970) 613-618.

[27] B. L. Sharma and K. C. Mittal, "Some new generating functions for Jacobi polynomials", Proc. Camb. Philos. Soc. 54 (1968) 691-694.

[28] H. M. Srivastava, "Hypergeometric functions of three variables", Ganita 15(2) (1964) 97-108.

[29] H. M. Srivastava, "Generalized Neumann expansions involving hypergeometric functions", Proc. Camb. Philos. Soc. 63 (1967) 428-429.

[30] H. M. Srivastava, "Some integrals representing triple hypergeometric functions", Rend. Circ. Mat. Palermo (2) 16(1-3) (1967) 99-115.

[31] H. M. Srivastava and H. L. Manocha, A treatise on generating functions (Hasted Press (Ellis Horwood, Chichester), John Wiley and Sons, New York, Chichester, Brisbane and Toronto, 1984). 